\title{
NOTA SOBRE TOURRETTIA LAPPACEA (BIGNONIACEAE), UN NUEVO REGISTRO PARA EL OCCIDENTE DE MÉXICO
}

\author{
Ramón Cuevas-Guzmán 1 , Nora M. NúÑez-López, José G. Morales-Arias
}

Universidad de Guadalajara, Instituto Manantlán de Ecología y Conservación de la Biodiversidad, Centro Universitario de la Costa Sur, Av. Independencia Nacional 151, Autlán de Navarro, 48900 Jalisco, México.

1Autor para la correspondencia: rcuevas@cucsur.udg.mx

\section{RESUMEN}

Se registra por primera vez a Tourrettia lappacea para el occidente de México, ampliando en más de $1300 \mathrm{~km}$ su distribución geográfica conocida. Se proporciona información sobre su hábitat y fotografías que ilustran a la especie.

Palabras clave: Chiapas, disyunción geográfica, Jalisco, Tourrettia.

\begin{abstract}
We register for the first time the presence of Tourrettia lappacea in western Mexico, expanding with more than $1300 \mathrm{~km}$ its known geographic distribution. We also provide information on its habitat and include photographs of the species.
\end{abstract}

Key words: Chiapas, geographic disjunction, Jalisco, Tourrettia.

El occidente de México, como lo define McVaugh (1961), comprende los estados de Jalisco, Colima, Aguascalientes y parte de los de Nayarit, Durango, Zacatecas, Guanajuato y Michoacán. Se puede considerar esta región como una de las áreas florísticamente mejor estudiadas de la República Mexicana, ya que numerosas familias han sido tratadas en la Flora Novo-Galiciana (e.g. McVaugh, 1984, 2001) y la Flora de Jalisco (e.g. Cervantes, 1992; Carvajal y Acosta, 2010). Además, recientemente se ha publicado un catálogo de las plantas vasculares de Jalisco (Ramírez- 
Delgadillo et al., 2010). A pesar de lo anterior, ciertas zonas siguen siendo poco exploradas y conforme se intensifican las excursiones botánicas se encuentran nuevas especies y registros, con una marcada tendencia a que éstos provengan de especies con afinidad neotropical, conocidas del sur de México y de Centroamérica (CuevasGuzmán et al., 2008; Cuevas-Guzmán y Montero, 2011; Carrillo-Reyes et al., 2013). Recientemente, durante la realización de un inventario florístico en la zona de Villas de Cacoma, en el municipio de Villa Purificación en el estado de Jalisco, se encontró una planta que fue identificada como Tourrettia lappacea (L'Hér.) Willd. ex. L. f. Esta especie pertenece a la familia Bignoniaceae y es una hierba anual con frutos con espinas ganchudas, por lo cual a primera vista se podría confundir con una representante de Cucurbitaceae.

Las Bignoniaceae incluyen alrededor de 110 géneros y más de 800 especies de plantas leñosas, o rara vez herbáceas, con una distribución pantropical, aunque con una mayor concentración de especies en América tropical (Gentry, 1982, 2001). De acuerdo con la Comisión Nacional para el Conocimiento y Uso de la Biodiversidad (CONABIO, 2008), se han consignado 41 géneros y 91 especies para México, distribuidos principalmente en las regiones cálido húmedas de ambas vertientes. En el estado de Jalisco se conocen de la familia, incluyendo elementos nativos y cultivados, 18 géneros y 29 especies (Ramírez-Delgadillo et al., 2010). Varios de ellos tienen importancia para la construcción, así como para uso ornamental, por la calidad de su madera y la belleza de sus flores. Tourrettia es un género monotípico, con distribución conocida de Argentina al estado de Chiapas en México, por lo cual el presente registro la amplía en más de $1300 \mathrm{~km}$ en línea recta.

La comparación minuciosa de los ejemplares del estado de Jalisco con los del resto de su área de distribución depositados en los herbarios ENCB, IEB y MEXU, y con las descripciones disponibles en la literatura (Standley y Williams, 1974; Burger y Gentry, 2000; Gentry, 2001, 2009) permite observar que las plantas del occidente y sur de México no presentan diferencias con las de Centro y Sudamérica. Se presenta así la siguiente descripción de la especie.

Tourrettia lappacea (L’Hér.) Willd. ex L. f. Sp. Pl., ed. 4. 3(1): 263. 1800. Basiónimo: Dombeya lappacea L'Hér. Stirp. Nov. 1: 33, t. 17. 1785. Tipo: PERÚ. 1781, Dombey 391 (Fotoisotipo: P!).

Hierba anual trepadora, de 4-7 m de longitud; tallos huecos; hojas opuestas, biternadas, provistas de un zarcillo con ramificaciones que terminan en 2-3 uñas recurvadas, foliolos laterales de cada terna divididos hasta la base, membranosos, glabros, 
con los márgenes serrados, lámina de 1-4 cm de largo, de 0.5-2.5 cm de ancho, lámina del foliolo central de 2.5-7 cm de largo, de 1.2-3.5 cm de ancho, pecíolos huecos, 4-8 $\mathrm{cm}$ de largo; inflorescencias en racimos espiciformes, flores dimórficas, las terminales por lo común estériles y caedizas, con el cáliz rojo escarlata, las fértiles con el cáliz tubular de 14-17 mm de largo, de 4-5 mm de ancho, de color verde, con los lóbulos rojo escarlatas, glandular pubescentes; corola tubular galeada, ligeramente geniculada en su base, de 20-23 mm de largo, de 4-5 $\mathrm{mm}$ de ancho en su ápice, verde-amarillenta, glandular pubescente; estambres didínamos, epipétalos, los filamentos de 7-10 $\mathrm{mm}$ de largo, de 0.7-0.9 mm de ancho, tecas divaricadas, de 1-2.5 mm de largo, glabras; ovario bicarpelar, de 2.4-3 mm de largo, de $1.5 \mathrm{~mm}$ de ancho, cubierto de tricomas uncinados, protegido por un anillo cupuliforme; infrutescencias péndulas, hasta de $28 \mathrm{~cm}$ de largo; cápsula ovoide u oblongo-ovoide, de 3-4 cm de largo, de 1.5-2 cm de grueso, bivalvada, con dehiscencia apical, las valvas algunas veces abriendo hasta cerca de la mitad, equinada, con espinas gruesas y uncinadas hasta de $15 \mathrm{~mm}$ de largo y otras más cortas y agudas de 3-6 mm de largo; semillas planas, de 6-7 mm de largo, de 4-5 $\mathrm{mm}$ de ancho, de color castaño oscuro, rodeadas por una ala angosta y membranosa (Fig. 1; un dibujo de la especie puede encontrase en Gentry, 2009, p. 414).

Ejemplares examinados: MÉXICO. Chiapas: Ángel Albino Corzo, Sierra de Soconusco, de la Finca Liquidámbar a El Triunfo, 9/XI/1945 (fl), Hernández X. y Sharp 424 (MEXU); cerca de Rancho Viejo de la Finca Prusia, 23/I/1968 (fl y fr), A. Shilom Ton 3618 (ENCB); $1 \mathrm{~km}$ al E de la Finca Prusia, 13/XI/1989 (fl), ReyesGarcía 1417 (MEXU); Chicomuselo, $2 \mathrm{~km}$ al O de Chicomuselo camino a Piedra Labrada, 18/XI/1984 (fl y fr), Martinez et al. 8681 (IEB, MEXU); Motozintla de Mendoza, 9 millas al SO de Motozintla de Mendoza, 6/XI/1980 (fl y fr), Fryxell y Lott 3330 (ENCB, MEXU); 4 km arriba de Tolimán camino a Motozintla, 22/ XI/1980 (fl), Breedlove 47668 (MEXU); Siltepec, Barranca Honda, X-XI/1940 (fl), Matuda 4044 (MEXU); Unión de Juárez, Col. 11 de abril, 16/II/1985 (fl), Ventura y López 1152 (IEB); Mt. Tacaná, VIII/1938 (fl), Matuda 2434 (MEXU). Jalisco: Villa Purificación, Villas de Cacoma, 19²9'23.5" N y 104³3'26.8" O; 1050-1100 m s.n.m.; 09/XII/2009 (fl y fr), Rodríguez y Morales 57 (ZEA); 19/III/2011 (fr), Cuevas et al. 10306 (ZEA); 8/XII/2012 (fl), Cuevas et al. 10900 (ZEA).

La familia Bignoniaceae está bien estudiada en México, pues ha sido tratada para las Floras de Veracruz (Gentry, 1982), del Bajío y Regiones Adyacentes (Rzedowski y Calderón de Rzedowski, 1993), de Guerrero (Martínez y Diego-Pérez, 2007), del Valle de Tehuacán-Cuicatlán (Martínez y Ramos, 2012) por lo cual sorprende que Tourrettia 

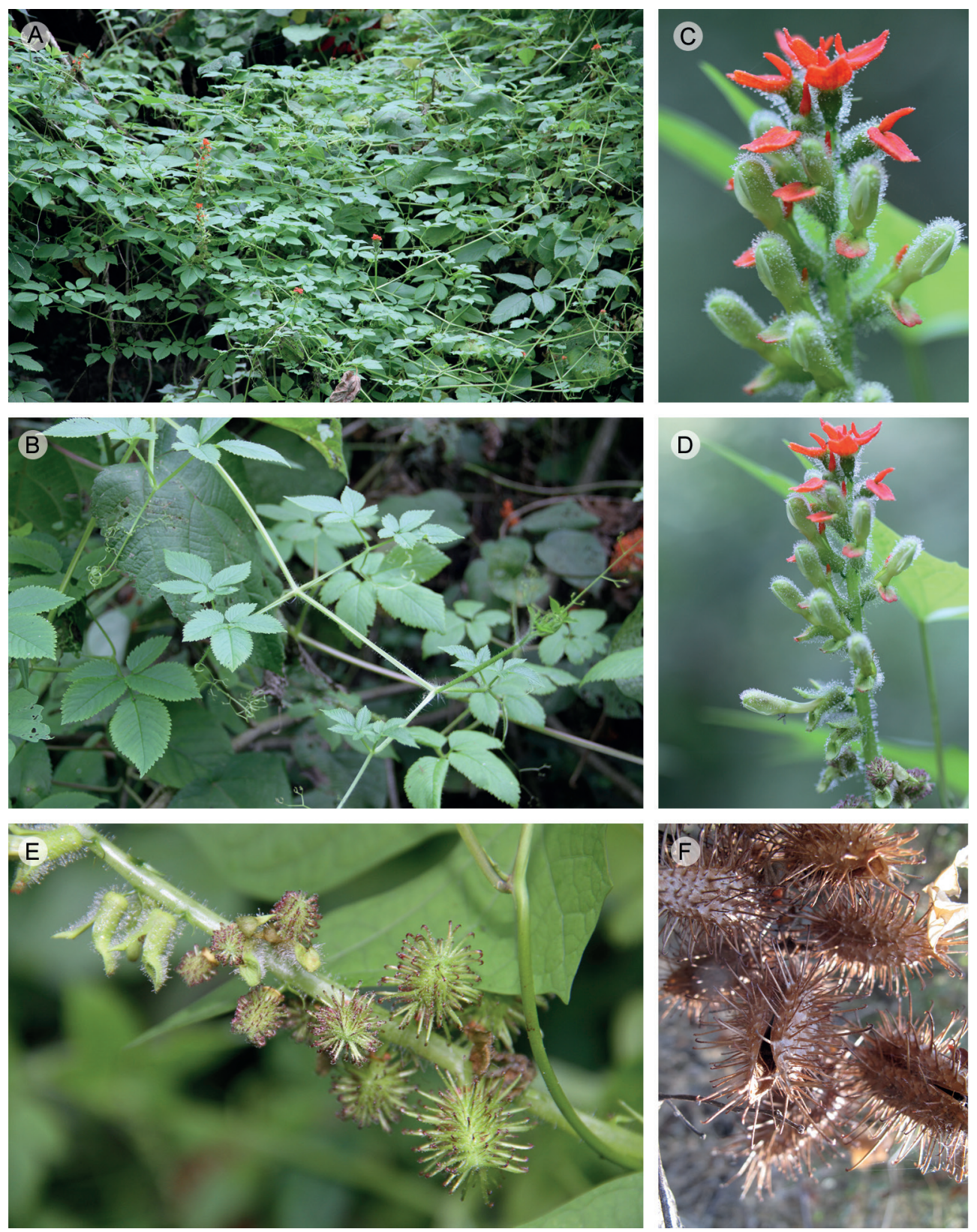

Fig. 1. Tourrettia lappacea. A. hábito de la especie; B. hojas, folíolos y ramificación de los tallos; C y D. flores funcionales con cáliz y corola, las estériles únicamente con cáliz y ubicadas en la parte superior de la inflorescencia; E. frutos jóvenes; F. frutos maduros en los que se observa la dehiscencia apical y las espinas uncinadas. 
lappacea solo haya sido registrada para Chiapas (Standley y Williams, 1974; Gentry, 1982). La revisión de ejemplares de esta especie en los principales herbarios nacionales da cuenta de una pobre recolección de especímenes en México.

Distribución y hábitat: De acuerdo con datos de literatura, Tourrettia lappacea crece en altitudes de 650-2 $200 \mathrm{~m}$, en bordes de bosques tropicales húmedos, así como en áreas abiertas dentro de los mismos (Standley y Williams, 1974; Burger y Gentry, 2000; Gentry, 2001, 2009). Las recolectas en que se basa el presente registro se realizaron al lado de una brecha que corre paralela al margen de un arroyo, donde la población presenta una extensión de unos $200 \mathrm{~m}$ de largo por $10 \mathrm{~m}$ de ancho, en las coordenadas $19^{\circ} 49^{\prime} 23.5^{\prime \prime} \mathrm{N}$ y $104^{\circ} 33^{\prime} 26.8^{\prime \prime} \mathrm{O}$, a una altitud de $1050-1100 \mathrm{~m}$, área alrededor de la cual se observaron especies de bosque tropical caducifolio y subcaducifolio como Annona reticulata L., Canna indica L., Centrosema sagittatum (Willd.) Brandg. ex Riley, Cleome speciosa Ralf., Coussapoa purpusii Standl., Croton draco Schltdl. \& Cham., Erythrina lanata Rose subsp. occidentalis (Standl.) Krukoff \& Barneby, Euphorbia pulcherrima Willd. ex Klotzsch, Hedyosmum mexicanum C. Cordem., Ipomoea santillanii O' Donell, Juglans major (Torr.) A. Heller var. glabrata W. E. Manning, Lysiloma acapulcense (Kunth) Benth., Otatea acuminata (Munro) C. E. Calderón \& Soderstr. y Periptera macrostelis Rose, entre otras.

Fenología: En Chiapas florece de agosto a febrero y fructifica de noviembre a febrero, mientras que en el occidente de México florece de septiembre a diciembre y fructifica de noviembre a marzo.

El hallazgo de Tourrettia lappacea en el occidente de México podría ser interpretado como una ampliación geográfica reciente del taxon. Sus frutos capsulares con espinas gruesas y uncinadas, con septos abriendo hasta cerca de la mitad de la longitud y sus semillas aladas, se identifican como rasgos asociados a un patrón de dispersión por epizoocoría y anemocoría. Aunque es difícil que un evento de larga distancia ocurra dejando huecos de distribución tan amplios, no es imposible, como ya se ha planteado para otros táxones como Calceolaria dichotoma (Padilla-Velarde y Cuevas-Guzmán, 2010). De hecho existe un conjunto de especies de repartición continua desde Sudamérica hasta México que son principalmente elementos de vegetación secundaria y de lugares perturbados. Varios de ellos alcanzan su límite de distribución boreal en Chiapas y Oaxaca y algunos otros llegan hasta Veracruz. En menor cantidad existen táxones que alcanzan el occidente de México por la vertiente pacífica (Rzedowski, 1978). Sin embargo, para el caso de Tourrettia lappacea no se 
puede descartar la posibilidad de que haya sido el hombre quien en tiempos recientes haya contribuido a su dispersión, pues se sabe que en la década de los sesenta del siglo anterior, se transportó maquinaria forestal procedente de Chiapas a Villas de Cacoma, Jalisco, para ser utilizada en la apertura de caminos y para la corta de árboles (Salvador Brambila Íñiguez, com. per.). Por el síndrome de dispersión de la especie, su preferencia por áreas perturbadas y la capacidad observada para la producción de frutos y semillas en el occidente de México, se espera que el taxon en el futuro ampliará su área de distribución en esta región.

\section{AGRADECIMIENTOS}

Al personal de las colecciones MEXU, ENCB, IEB, IBUG, CHIP, por facilitar el acceso a la consulta del material de sus herbarios. Al Consejo Estatal de Ciencia y Tecnología del estado de Jalisco por el apoyo brindado a través de los proyectos: Estructura, diversidad y reservorios de carbono de bosques de cañadas en el Pacífico Mexicano y Museo regional de Ciencia y Tecnología en la costa sur del estado de Jalisco. Al Dr. Emmanuel Pérez Calix por su apoyo en la consulta de material de herbario depositado en IEB.

\section{LITERATURA CITADA}

Burger, W. y A. Gentry. 2000. Family 194. Bignoniaceae. Flora Costaricensis. Fieldiana, Bot., n.s. 41: 77-161.

Carrillo-Reyes, P., E. Sahagún-Godínez y G. Ibarra-Manríquez. 2013. The genus Disciphania (Menispermaceae: Tinosporoideae, Tinosporeae) in Mexico. Brittonia 65(4): 439-451.

Carvajal, S. y L. L. Acosta. 2010. Muntingiaceae. Flora de Jalisco y Áreas Colindantes 24: 1-14.

Cervantes, N. 1992. La familia Malvaceae en el estado de Jalisco. Colección Flora de Jalisco 3: $1-393$.

CONABIO. 2008. Dicotiledóneas. In: Ocegueda S. y J. Llorente-Bousquets (coords.). Catálogo taxonómico de especies de México. Conocimiento Actual de la Biodiversidad. Capital Natural de México. Vol. I. Comisión Nacional para el Conocimiento y Uso de la Biodiversidad. México, D.F., México. http://www.biodiversidad.gob.mx/pais/ pdf/catalogos.zip

Cuevas-Guzmán, R., E. García-Moya, J. A. Vázquez-García y N. M. Núñez-López. 2008. Estructura poblacional y relaciones ambientales del árbol tropical Nectandra rudis (Lauraceae), una especie rara en el occidente de México. Rev. Biol. Trop. 56: 247-256. 
Cuevas-Guzmán, R. y J. C. Montero. 2011. Nuevo registro de Cestrum mortonianum (Solanaceae) y descripción de una variedad en la vertiente pacífica de México. Acta Bot. Mex. 95: 37-43.

Gentry, A. H. 1982. Bignoniaceae. Flora de Veracruz 24: 1-122.

Gentry, A. H. 2001. Bignoniaceae. In: Stevens, W. D., C. Ulloa U., A. Pool y O. M. Montiel (eds.). Flora de Nicaragua. Missouri Botanical Garden Press. pp. 403-428.

Gentry, A. H. 2009. Bignoniaceae. Flora de Colombia 25: 1-462.

Martínez, T. y N. Diego-Pérez. 2007. Bignoniaceae. Flora de Guerrero 29: 1-101.

Martínez, E. y C. H. Ramos. 2012. Bignoniaceae. Flora del Valle de Tehuacán-Cuicatlán 104: 1-58.

McVaugh, R. 1961. Euphorbiaceae novae Novo-Galicianae. Brittonia 13: 145-205.

McVaugh, R. 1984. Compositae. In: Anderson, W. R. (ed.). Flora Novo-Galiciana. Vol. 12. The University of Michigan Press. Ann Arbor, Michigan. 1157 pp.

McVaugh, R. 2001. Ochnaceae to Loasaceae. In: Anderson W. R. (ed.). Flora NovoGaliciana. Vol. 17. The University of Michigan Press. Ann Arbor, Michigan. 751 pp.

Padilla-Velarde, E. y R. Cuevas-Guzmán. 2010. Calceolaria dichotoma subsp. colimana (Calceolariaceae), una subespecie nueva del occidente de México. Acta Bot. Mex. 91: 95-102.

Ramírez-Delgadillo, R., O. Vargas P., H. J. Arredondo N., M. Cedano M., R. González T., L. M. González V., M. Harker, L. Hernández L., R. E. Martínez G., J. A. Pérez de la Rosa, A. Rodríguez C., J. J. Reynoso D., L. M. Villarreal de Puga y J. L. Villaseñor R. 2010. Catálogo de plantas vasculares de Jalisco. Prometeo Editores, S.A. de C.V. Guadalajara, Jalisco, México. 143 pp.

Rzedowski, J. 1978. Vegetación de México. Limusa, México, D.F., México. 432 pp.

Rzedowski, J. y G. Calderón de Rzedowski. 1993. Familia Bignoniaceae. Flora del Bajío y de Regiones Adyacentes 22: 1-44.

Standley, P. C. y L. O. Williams. 1974. Bignoniaceae. Flora de Guatemala. Fieldiana, Bot. 24: $153-232$. 